\title{
B. Mérenne-Schoumaker, Géographie de l'énergie
}

Paris : Belin, 2007

\section{Michel Deshaies}

\section{OpenEdition \\ Journals}

Édition électronique

URL : http://journals.openedition.org/rge/1623

DOI : $10.4000 /$ rge. 1623

ISSN : 2108-6478

Éditeur

Association des géographes de l'Est

Édition imprimée

Date de publication : 1 juin 2007

ISSN : 0035-3213

\section{Référence électronique}

Michel Deshaies, «B. Mérenne-Schoumaker, Géographie de l'énergie », Revue Géographique de l'Est [En ligne], vol. 47 / 3 | 2007, mis en ligne le 08 novembre 2011, consulté le 23 septembre 2020. URL: http://journals.openedition.org/rge/1623 ; DOI : https://doi.org/10.4000/rge.1623

Ce document a été généré automatiquement le 23 septembre 2020.

Tous droits réservés 


\section{B. Mérenne-Schoumaker, Géographie de l'énergie}

Paris : Belin, 2007

Michel Deshaies

\section{RÉFÉRENCE}

B. Mérenne-Schoumaker, Géographie de l'énergie, Paris : Belin, 2007, 271p. 
1 À l'heure où la croissance de la consommation énergétique mondiale accroît la pression sur les ressources de la planète et sur l'environnement global, le livre de Bernadette Mérenne-Schoumaker illustre magnifiquement ce que la géographie peut apporter à la compréhension des grands problèmes contemporains. En ce sens, il participe au retour de la géographie à la place qui lui revient naturellement comme discipline d'information $\mathrm{du}$ citoyen sur un sujet beaucoup plus souvent fréquenté par d'autres. En effet, si l'énergie est devenue depuis quelques années l'un des grands sujets de débat dans la société, force est de constater que dans la masse énorme et en forte croissance des publications consacrées chaque année à cette question,

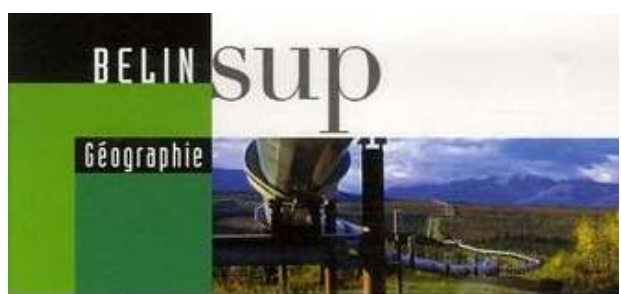

\section{Géographie} de l'énergie

Acteurs, lieux et enjeux

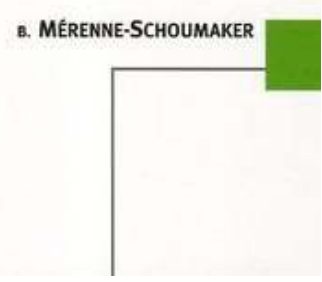
la part des géographes est pour le moins modeste. C'est pourquoi il convient de saluer cette publication parue précisément l'année où la thématique énergétique est au cœur de l'actualité et a été retenue comme thème du Festival International de Géographie à St-Dié. Il faut en effet souligner l'intérêt et la spécificité de l'approche géographique de l'énergie que, dès le premier chapitre, l'auteur définit comme une "clé de lecture des territoires ». L'énergie est en effet une des grandes ressources constituant une contrainte, ou au contraire une «opportunité pour le développement d'un territoire » dont l'organisation spatiale est plus ou moins largement influencée par «les modalités de son approvisionnement énergétique ». Plus largement, la géographie de l'énergie apporte une contribution originale aux débats concernant la mondialisation croissante des échanges, l'étalement urbain résultant d'une énergie longtemps bon marché et le développement durable.

2 Sous un volume relativement modeste, l'ouvrage articulé en 8 chapitres fait véritablement le tour de la question, tant en ce qui concerne les différentes sources de production que les problématiques de consommation et d'utilisation de l'énergie, en en montrant les dimensions techniques, économiques, géopolitiques et environnementales. Parmi les nombreux mérites de ce manuel, il convient de souligner qu'il présente au lecteur une information solide et abondante, mais néanmoins très accessible, grâce à un souci pédagogique omniprésent. En effet, chaque chapitre est illustré de cartes et de schémas, le plus souvent extraits de la documentation fournie par les sociétés de production d'énergie, si bien que l'étudiant le moins averti peut assimiler facilement la formation du charbon et des hydrocarbures, ou le fonctionnement des centrales thermiques, hydroélectriques ou nucléaires. De plus, l'ouvrage comporte une bibliographie abondante, avec une sélection d'ouvrages classiques et de nombreuses références de sites internet fournissant les données statistiques indispensables, ainsi que des dossiers d'information.

3 L'ouvrage commence logiquement par l'étude des ressources permettant de produire de l'énergie (chapitres 2 et 3), avant d'étudier la question de la consommation, des 
marchés et des échanges internationaux dans ses dimensions à la fois économiques et géopolitiques (chapitres 4,5 et 6 ). Il se termine par une présentation des conséquences environnementales de l'utilisation des différentes sources d'énergie (chapitre 7), puis de l'avenir des différentes ressources énergétiques, questions on ne peut plus actuelles. Au-delà d'une information toujours très rigoureuse, où les données statistiques permettent de bien mesurer les potentialités et les enjeux liés à l'exploitation et à l'utilisation des différentes ressources énergétiques, chaque chapitre comporte des études de cas avec des questionnements problématisés comme « les biocarburants : une nouvelle commodité ", "les richesses pétrolières et gazières de l'Asie centrale ", ou encore « le peak-oil ou l'annonce de la fin du pétrole». Ces documents qui ont vocation à faire réfléchir le lecteur, l'invitent ainsi à participer au débat que suscitent bien des problématiques énergétiques.

En effet, peu de questions suscitent aujourd'hui plus de controverses que celle de l'approvisionnement énergétique actuel et futur, ainsi que celle de l'impact environnemental de l'exploitation des différentes sources d'énergie. Toutes ces questions sont présentées avec beaucoup de rigueur et d'honnêteté. L'auteur passe ainsi en revue les avantages et les inconvénients pour l'approvisionnement énergétique et l'environnement des différentes sources d'énergie, tout en soulignant les potentialités du progrès technique. Ce faisant, le lecteur dispose avec ce manuel des éléments essentiels permettant de prendre la mesure des grands enjeux énergétiques : qu'il s'agisse de l'estimation de l'importance des réserves en énergie fossile, des inégalités Nord-Sud d'accès à l'énergie ou de l'évolution des émissions de gaz à effet de serre et leur impact climatique, tous ces sujets sont éclairés par les données rassemblées par l'auteur qui montre aussi le recul et la prudence nécessaires concernant les scénarios possibles pour l'avenir de l'approvisionnement énergétique.

On ne peut donc que recommander chaudement la lecture de ce manuel de référence qui donne toutes les clés indispensables à la compréhension des problématiques énergétiques, non seulement aux géographes, mais plus généralement au citoyen désirant disposer d'un éclairage objectif sur ce grand sujet d'actualité.

\section{AUTEURS}

\section{MICHEL DESHAIES}

CERPA, Université Nancy 2 